\title{
Comparative virulence of Scedosporium species in animal models
}

\begin{abstract}
Scedosporium species are an emerging opportunist group of fungi that have been found to cause infections in both immunocompetent and non-immunocompetent individuals. The infections are not regularly distributed among different countries of the world either because of improper identification or other geographical reasons. Strange as it may, disseminated systemic infections have only been reported in some specific countries. We used a mouse model of disseminated infection to assess if strains from Nigeria were virulent and compared it to a few other strains from other countries. S. apiospermum isolated from Nigeria were clearly less virulent than those obtained elsewhere. This may be the reason why this group of fungi has not been associated with specific clinical problems in Nigeria in pa'rticular and Africa in general.
\end{abstract}

Keywords: Scedosporium, inocula, virulence, fungi.

[Braz J Infect Dis 2010;14(3):271-276] @Elsevier Editora Ltda.

\section{INTRODUCTION}

Scedosporium species are filamentous fungi that belong to the class Euascomycetes, of the order Microascales, and family Microascaceae. Until recently, the genus Scedosporium contained two species: Scedosporium apiospermum and Scedosporium prolificans. Other new species such as $S$. dehoogi and $S$. auranticum have been proposed and apparently accepted. ${ }^{1}$ Scedosporium species were originally known to be soil-borne organisms, but they have emerged recently as opportunistic pathogens in both immunocompetent and immunocompromised patients. ${ }^{2-3}$ They are known to be distributed worldwide. ${ }^{4-5}$ Nevertheless, information on the species does not seem balanced, as there are very few reports, from Africa. Moreover, it is possible that the virulence of strains could vary from one geographical location to another. Owing to the apparent threat posed by this species, particularly because of its poor response to conventional antifungals, ${ }^{6-7}$ we conducted this study to compare the virulence of some species isolated from Nigeria and those from other geographical areas. Recently, Capilla and Guarro $^{8}$ proposed a model of disseminated infection of this group of fungi using neutropenic guinea pigs and observed some strain-dependent differences. They also achieved total mortality with mean survival times of about 3-4 days with highest inocula sizes of $2 \times 10^{6}$ and $7 \times 10^{5}$. When they used the lowest inoculum of $3 \times 10^{5}$, mortality was reduced to $50 \%$ for one strain and $100 \%$ for the other, and the mean survival times were 12.5 and 5.25 days, respectively. Brain and kidneys were extensively infected by both strains of Scedosporium used in that study. The authors therefore concluded that the mortality rate correlated with inoculum density, strain differences, and immune status. Thus, the variability in virulence observed among different strains of Scedosporium and recent reports of imported infections across so many countries indicate that more data on the virulence of a wide range of strains, especially from developing countries of Africa are clearly needed.

\section{MATERIAL AND METHODS}

\section{Virulence studies}

Isolates and preparation of inocula: Scedosporium isolates tested in this study were selected based on a DNA-DNA reassociation experiment, which separated Scedosporium apiospermum isolates into three groups identified as
Authors

Nweze EI ${ }^{1,2}$

Okafor $\mathrm{JI}^{1}$

${ }^{1}$ Department of

Microbiology, University of Nigeria, Nsukka, Nigeria.

${ }^{2}$ Department of

Dermatology, Case Western Reserve University,

Cleveland, OH 44106, USA.
Submitted on: 09/05/2009 Approved on: 12/22/2009

Correspondence to:

Nweze EI

Department of

Microbiology, University of Nigeria, Nsukka,

Nigeria

Phone: +234-80-68535841

E-mail: nwezemeka@

yahoo.com

We declare no conflict of interest. 
groups I to III. All the isolates were first cultured on potato dextrose agar (PDA) for 7-10 days at $30^{\circ} \mathrm{C}$. The inocula were prepared by flooding the surface of the agar plate with sterile saline, scraping the sporulating mycelium with a culture loop, and drawing up the resultant suspension with a sterile Pasteur pipette. The suspensions were then filtered once through sterile gauze to remove hyphae. The number of conidia in the suspension was counted with a haemocytometer, adjusted to $\left(8 \times 10^{5}\right)-\left(1 \times 10^{6}\right)$ conidia/mL and verified by plating dilutions of the suspension on PDA plates.

Animals: Male mice with a mean weight of $32 \mathrm{~g}$ were used. These animals were housed eight per cage in standard boxes with corncob bedding and free access to food and water.

Infection, histopathology and statistical analysis: Inoculum suspensions $(0.2 \mathrm{~mL}$ of 106 conidia/mL) of each fungal strain were injected intravenously via the lateral tail vein in groups of eight mice. Mortality was recorded daily for 30 days. Mean survival time (MST) was estimated by the Kaplan-Meier method in the SPSS software (version 10) and compared among groups by the log-rank test. Representative samples of organs from these animals were fixed in $10 \%$ neutral buffered formaldehyde for 10 days, embedded in paraffin wax and automatically processed. Sections $(3 \mu \mathrm{m}$ in thickness) of the embedded tissues were stained with methenamine silver (Grocott) for light microscopy observations.

\section{Comparative infectivity of isolates by different routes}

The inocula were prepared as described above. However, 2 $\mathrm{x} 10^{6}$ conidia per $\mathrm{mL}$ concentration of cells were used and white male mice weighing 18-22 g were used. Two groups of 6 mice each were used per route of inoculation. They were injected with saline suspension of the isolates tested. The intraperitoneal and subcutaneous dose of inoculum was 0.5 $\mathrm{mL}$, while $0.2 \mathrm{~mL}$ was used for the intravenous injections via the lateral tail vein. Half the mice population was sacrificed after two weeks and the rest after 5 weeks. The lungs, liver, spleen, kidneys, diaphragm, intestine, brain, and stomach were examined for the presence of lesions. Bits of these organs and tissue were macerated and cultured on brain heart infusion agar (BHIA) slants supplemented with chloramphenicol $(0.05 \mathrm{mg} / \mathrm{mL})$ and incubated at $30^{\circ} \mathrm{C}$ for up to 4 weeks. Portions of the organs and tissues showing macroscopic lesions were digested in $10 \% \mathrm{KOH}$ and observed microscopically for the presence of fungal elements.

\section{RESULTS}

The result confirmed the virulence of Scedosporium species in mice. However, all the three S. apiopsermum group I isolates (SA1, SA80 and SA31) could not cause death in mice 30 days after inoculation, as $63 \%, 64 \%$ and $69 \%$ of the mice survived, respectively, after intravenous inocu- lation of the mice with each isolate through the lateral vein with $0.2 \mathrm{~mL}$ of $10^{6}$ conidia/ $\mathrm{mL}$ of each isolate (Figure 1). Similarly, out of the three isolates of S. apiospermum tested in group II, SA35 and SA68 produced similar virulence patterns, as $60 \%$ of mice inoculated with each isolate survived after 30 days postinfection. The third isolate in the group, SA157, was slightly less virulent, as $62 \%$ of the mice inoculated with prepared conidia survived after 30 days postinfection (Figure 2). In group III, two isolates (SA151 and SA166) showed a similar pattern of virulence with those in groups II and III by recording 59 and $63 \%$ survival after 30 days postinfection, respectively. The third isolate tested in group III, SA138, had a much lower virulence, as $90 \%$ of the mice survived 30 days postinfection (Figure 3). This isolate showed a significant deviation in virulence when compared to the other isolates in group III or the isolates tested in groups I and II. Five isolates of S. prolificans (SP1, SP11, SP13, SP14, and SP15) were tested in addition to typed Spanish control strain, FMR3569 (Figures $4,5)$. S. prolificans isolates were generally more virulent than S. apiospermum. For instance, 30 days postinfection of SP 1 , SP 13 , and SP 15 isolates, $10 \%, 35 \%$, and $30 \%$ of the mice, respectively, survived. For SP11 and SP14 isolates, $25 \%$ and $9 \%$ of mice, respectively, survived 30 days postinfection. The $S$. prolificans Spanish control strain was significantly more virulent than any of the S. prolificans isolates. After nine days postinfection, all mice inoculated with the control strain died. The logrank test of statistical package for social sciences (SPSS version 10), used to analyze the result, placed all isolates tested into four classes of virulence: high, intermediate, low, and lower. The control strain was the only isolate with high virulence. Additionally, SP1 and SP14 had intermediate virulence, while the rest of the SP isolates were classified as isolates with low virulence. All SA isolates had lower virulence (Table 1).

Figure 1: Survival of mice infected with SA group I isolates by intravenous inoculation through the lateral vein with (0.2 mL of $10^{6}$ conidia/mL).

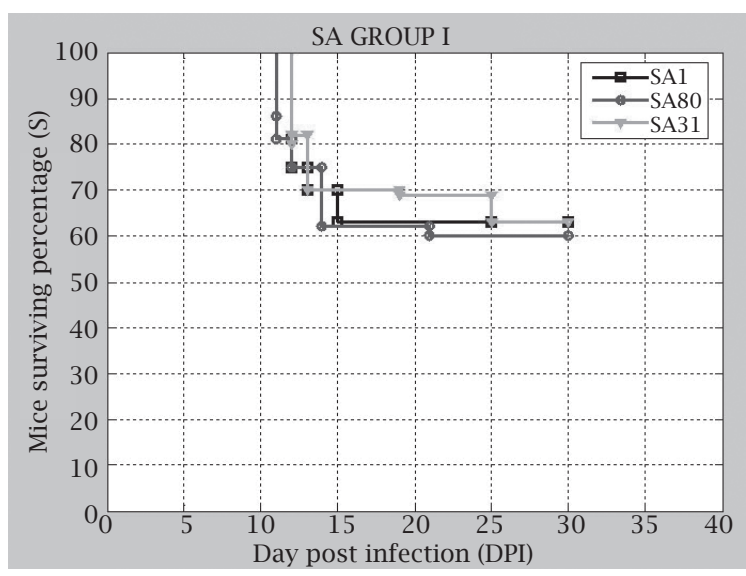


Figure 2: Survival of mice infected with SA group II isolates by intravenous inoculation through the lateral vein with $\left(0.2 \mathrm{~mL}\right.$ of $10^{6}$ conidia $\left./ \mathrm{mL}\right)$ conidia.

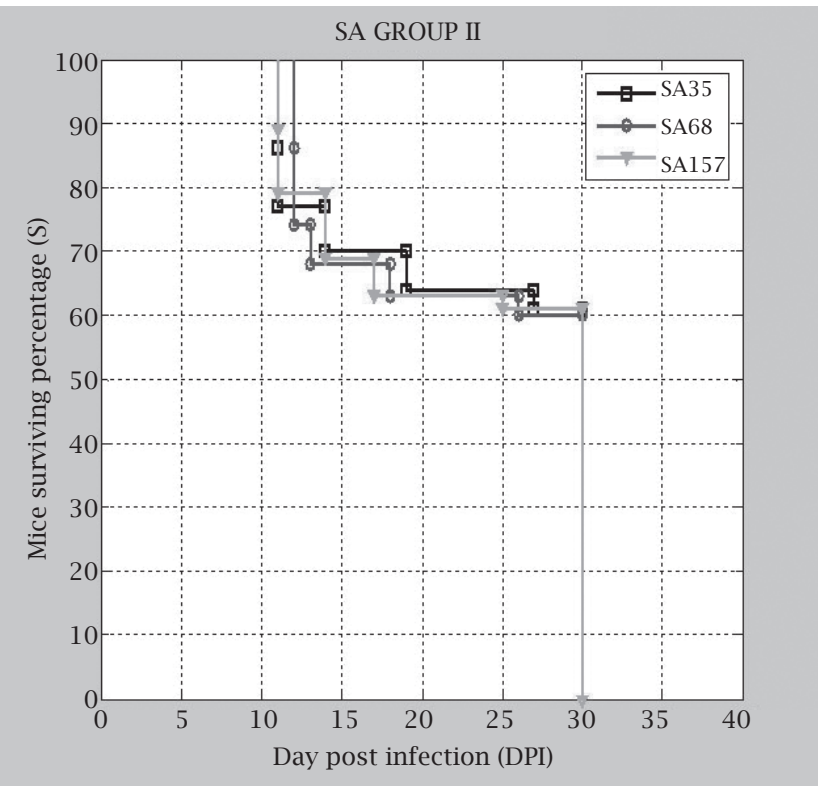

Figure 3: Survival of mice infected with SA group III isolates by intravenous inoculation through the lateral vein with $\left(0.2 \mathrm{~mL}\right.$ of $10^{6}$ conidia $\left./ \mathrm{mL}\right)$ conidia.

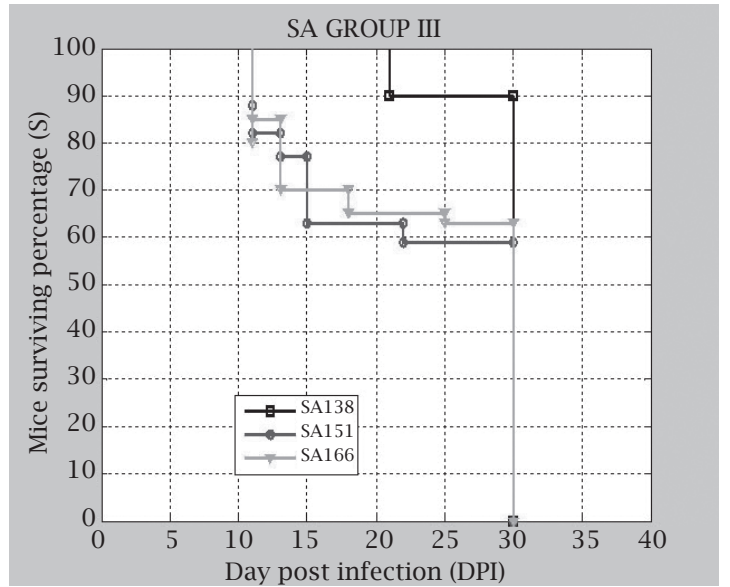

Figure 4: Survival of mice infected with SP isolates: SP1, 13 and 15 by intravenous inoculation through the lateral vein with $\left(0.2 \mathrm{~mL}\right.$ of $10^{6}$ conidia/mL $)$ conidia.

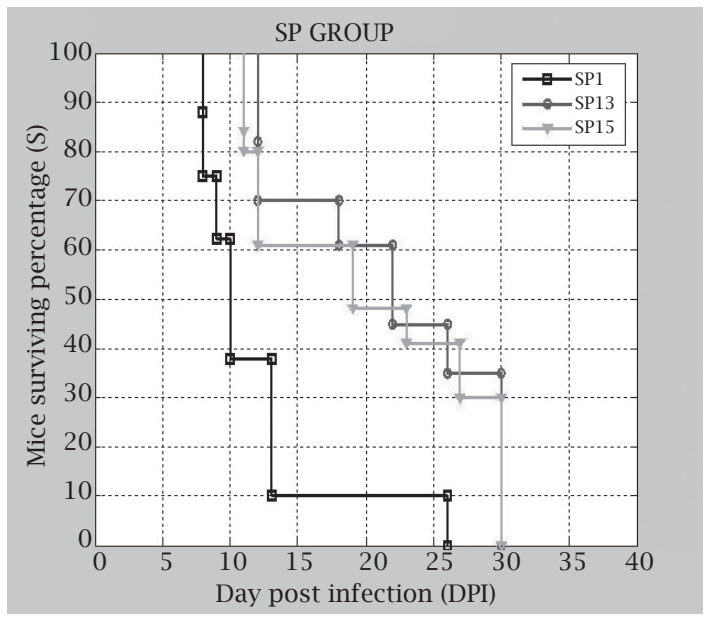

Figure 5: Survival of mice infected with SP isolates: SP11, 14 and the control Spanish strain FMR 3569 by intravenous inoculation through the lateral vein with $(0.2 \mathrm{~mL}$ of $10^{6}$ conidia/mL) conidia.



Table 1. Scedosporium species grouped according to their virulence in mice

\begin{tabular}{|c|c|c|c|c|c|}
\hline Species & ree of virulence & Isolate number & $\begin{array}{l}\text { Mean survival time } \\
\text { (MST) (days) (95\% CI) }\end{array}$ & Mortality & p-value \\
\hline \multirow[t]{3}{*}{ S. prolificans } & High & FMR 3569 & $7.8(5.7-8.9)$ & 100 & 0.0003 \\
\hline & Intermediate & SP14,SP1 & $12.94(10.72-13.81)$ & $90.1^{x}$ & - \\
\hline & Low & SP11,SP13,SP15 & $24.2(19.51-29.72)$ & 70 & 0.0009 \\
\hline S. apiospermum & Lower & $\begin{array}{c}\text { SA1,SA80,SA31,SA35,SA68, } \\
\text { SA157,SA138,SA151, SA166 } \\
\text { (Nigerian strains) }\end{array}$ & $27.13(23.90-29.20)$ & $26^{\#}$ & - \\
\hline
\end{tabular}

*, Significance versus the group with intermediate virulence in the log-rank test; *, Mean values for the nine isolates included in

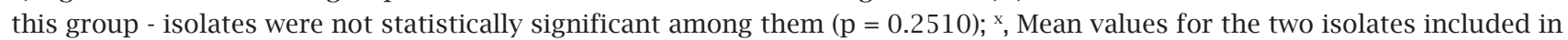
this group - isolates were not statistically different among them $(\mathrm{p}=0.1820)$. 


\section{Comparative infectivity of Scedosporium isolates}

In organs and tissues, the diameter of formed lesions ranged from 1 to $2.5 \mathrm{~mm}$. They were nodular. The formation of lesions depended on the route of inoculation and in some cases, the time of sacrifice. In intravenously infected animals, lesions were seen in the liver, kidney, lungs, spleen, and brain (Table 2). However, no lesions were formed in the stomach, intestine, and diaphragm of these animals. This observation differed from those mice injected intraperitoneally, especially with regard to lesion formation in similar organs. The ratio of mice showing positive recovery results for tissue culture to the number of mice showing positive results for presence of lesions in the organs was similar for most of Scedosporium isolates injected intravenously.

Table 2. Distribution of lesions in mice sacrificed two weeks (2W) and 5 weeks (5W) postinfection with saline suspended inocula of Scedoporium apiospermum and Scedosporium prolificans isolates via intravenous route (A) and intraperitoneal route (B)

\begin{tabular}{|c|c|c|c|c|c|c|c|c|}
\hline \multirow{2}{*}{$\begin{array}{l}\text { A } \\
\text { Isolates }\end{array}$} & \multicolumn{6}{|c|}{$\begin{array}{l}\text { Number of mice showing positive result of recovery of culture from tissue / } \\
\text { Number of mice showing positive result for presence of lesions in organs }\end{array}$} & \multirow[b]{2}{*}{$\begin{array}{l}\text { Diaphragm } \\
\text { 2W } 5 W\end{array}$} & \multirow[b]{2}{*}{$\begin{array}{l}\text { Brain } \\
2 W 5 W\end{array}$} \\
\hline & $\begin{array}{c}\text { Liver } \\
2 \mathrm{~W} 5 \mathrm{~W}\end{array}$ & $\begin{array}{l}\text { Kidney } \\
2 W 5 W\end{array}$ & $\begin{array}{l}\text { Lungs } \\
2 W 5 W\end{array}$ & $\begin{array}{l}\text { Spleen } \\
2 W 5 W\end{array}$ & $\begin{array}{c}\text { Stomach } \\
2 W 5 W\end{array}$ & $\begin{array}{c}\text { Intestine } \\
2 \mathrm{~W} 5 \mathrm{~W}\end{array}$ & & \\
\hline \multirow[t]{2}{*}{ SA1 } & $3 / 3$ & $3 / 3$ & $3 / 3$ & $3 / 3$ & $0 / 0$ & $0 / 0$ & $0 / 0$ & $3 / 3$ \\
\hline & $3 / 3$ & $3 / 3$ & $3 / 3$ & $3 / 3$ & $0 / 0$ & $0 / 0$ & $0 / 0$ & $3 / 3$ \\
\hline \multirow[t]{2}{*}{ SA35 } & $3 / 3$ & $3 / 3$ & $3 / 3$ & $3 / 1$ & $0 / 0$ & $0 / 0$ & $0 / 0$ & $3 / 3$ \\
\hline & $3 / 3$ & $3 / 3$ & $3 / 3$ & $3 / 3$ & $0 / 0$ & $0 / 0$ & $0 / 0$ & $3 / 3$ \\
\hline \multirow[t]{2}{*}{ SA68 } & $3 / 3$ & $3 / 2$ & $3 / 3$ & $3 / 3$ & $0 / 0$ & $0 / 0$ & $0 / 0$ & $3 / 3$ \\
\hline & $3 / 3$ & $3 / 3$ & $3 / 3$ & $1 / 0$ & $0 / 0$ & $0 / 0$ & $0 / 0$ & $3 / 3$ \\
\hline \multirow[t]{2}{*}{ SP1 } & $3 / 3$ & $3 / 3$ & $3 / 3$ & $3 / 0$ & $0 / 0$ & $0 / 0$ & $0 / 0$ & $3 / 3$ \\
\hline & $3 / 2$ & $3 / 3$ & $3 / 3$ & $3 / 2$ & $0 / 0$ & $0 / 0$ & $0 / 0$ & $2 / 3$ \\
\hline \multirow[t]{2}{*}{ SP15 } & $3 / 3$ & $3 / 3$ & $3 / 3$ & $2 / 0$ & $0 / 0$ & $0 / 0$ & $0 / 0$ & $3 / 2$ \\
\hline & $3 / 3$ & $3 / 3$ & $3 / 3$ & $3 / 3$ & $0 / 0$ & $0 / 0$ & $0 / 0$ & $3 / 3$ \\
\hline \multicolumn{9}{|l|}{ B } \\
\hline \multirow[t]{2}{*}{ SA1 } & $3 / 3$ & $3 / 3$ & $3 / 3$ & $3 / 0$ & $3 / 0$ & $1 / 1$ & $0 / 0$ & $3 / 0$ \\
\hline & $3 / 3$ & $3 / 3$ & $3 / 3$ & $3 / 1$ & $3 / 1$ & $3 / 3$ & $0 / 0$ & $3 / 0$ \\
\hline \multirow[t]{2}{*}{ SA35 } & $3 / 3$ & $3 / 3$ & $3 / 3$ & $3 / 0$ & $3 / 2$ & $2 / 2$ & $1 / 0$ & $2 / 3$ \\
\hline & $3 / 3$ & $3 / 3$ & $3 / 3$ & $3 / 0$ & $2 / 1$ & $2 / 2$ & $0 / 0$ & $3 / 2$ \\
\hline \multirow[t]{2}{*}{ SA68 } & $3 / 3$ & $3 / 3$ & $3 / 3$ & $3 / 0$ & $2 / 2$ & $3 / 0$ & $3 / 2$ & $3 / 2$ \\
\hline & $3 / 3$ & $3 / 2$ & $2 / 3$ & $3 / 1$ & $2 / 0$ & $2 / 2$ & $3 / 1$ & $3 / 3$ \\
\hline \multirow[t]{2}{*}{ SP1 } & $3 / 2$ & $3 / 3$ & $3 / 0$ & $3 / 3$ & $3 / 2$ & $3 / 3$ & $2 / 0$ & $2 / 0$ \\
\hline & $3 / 3$ & $3 / 2$ & $3 / 1$ & $3 / 2$ & $3 / 3$ & $3 / 2$ & $3 / 3$ & $1 / 0$ \\
\hline \multirow[t]{2}{*}{ SP15 } & $3 / 3$ & $3 / 3$ & $3 / 2$ & $3 / 3$ & $3 / 3$ & $3 / 3$ & $2 / 2$ & $2 / 2$ \\
\hline & $3 / 2$ & $3 / 1$ & $3 / 3$ & $3 / 3$ & $3 / 0$ & $3 / 1$ & $3 / 2$ & $2 / 1$ \\
\hline
\end{tabular}




\section{DISCUSSION}

Fienberg ${ }^{9}$ and Ajello ${ }^{10}$ were the first authors that documented studies involving the virulence of Scedosporium species in animal models. They independently used different routes of inoculation to inject the animals and found that virulence appeared low when intraperitoneal inoculation was used. Each author was able to isolate the organism again several weeks after inoculation, but could not find any sign of injury to visceral organs of animals. Several years later, Reyes ${ }^{11}$ and Schmitt et al. ${ }^{12}$ used the same procedure of Ajello, ${ }^{10}$ but they were unable to reproduce these results. The reason for this observation could not be immediately ascertained until years later when Bell ${ }^{13}$ indicated that human isolates of S. apiospermum are considerably more virulent than soil or manure strains. Thus, it was then concluded that the inability to reproduce the results of Ajello could be probably explained by the use of strains with different degrees of virulence, as virulence of Scedosporium species is strain dependent. This was the main motivation for our study.

Figures 1 to 4 compare the virulence of strains isolated from different clinical and non-clinical sources in a murine model. All S. apiospermum strains tested in the study were clearly less virulent (Mean survival time (MST) of $27 .{ }^{13}$ days and mean mortality rate of $26 \%$ ) than those of any of the other three virulence groups of $S$. prolificans (MST 7.80, 12.94, and 24.20 days and mean mortality rates of $100.0 \%, 90.1 \%$, and $70.0 \%$ for the high, intermediate, and low virulence groups, respectively (Table 1 ). Similar results on the high virulence of $S$. prolificans compared to S. apiospermum were also observed by other authors in a similar murine model. ${ }^{14,15}$ Although selected isolates were used in addition to only one reference typed Spanish strain due to the complexity and cost of testing so many animals, the result suggests that the Spanish control strain was more virulent than any of the Scedosporium apiospermum species isolated from Nigeria. It is possible that when more isolates are tested, this observation may not stand. Ortoneda et al. ${ }^{14}$ compared Spanish isolates to isolates recovered from the USA and concluded that they were not more virulent than those from the USA. However, they could not explain the reason why disseminated infections by these fungi are more frequent in Spain than in the USA. Likewise, disseminated Scedosporium infection is not very common in Nigeria as well. It is likely that the clinical differences observed could be due to other factors, such as the different genetic susceptibility of people from different geographical regions. This study also demonstrates that there are no differences in virulence between clinical and non-clinical strains and that any isolate from non-clinical or clinical source can cause severe infections in a patient with predisposing factors. This seems to be in agreement with observations by Ortoneda et al. ${ }^{15}$ It is also clear from the results that strains with different virulent patterns can exist in the same region. This is supported by a similar observation by Bell ${ }^{13}$ who found differences in virulence between a strain from a patient with subcutaneous infection and a strain from the environment. The distribution of lesions in mice sacrificed two weeks $(2 \mathrm{~W})$ and 5 weeks $(5 \mathrm{~W})$ postinfection (Table 2) with saline suspended inocula of some selected isolates of $S$. apiospermum and S. prolificans via the intravenous route (A) and intraperitoneal route (B) shows that the formation and persistence of lesions in organs and tissues depended on the route of inoculation and time of sacrifice. Lesions were seen in the liver, spleen, kidney, stomach, intestine, diaphragm, brain, and lungs of the intraperitoneallly infected mice, while no lesions were seen in the stomach, intestine, and diaphragm of intravenously inoculated animals. An interesting observation is the ability of all Scedosporium strains studied to produce lesions in the brain. This confirms the predilection of these fungi for brain tissues, which is more often reported in patients of near-drowning. The ability of Scedosporium species to cause invasive infection is also justified by its ability to attack all the visceral organs, particularly when it initiates infection through intravenous route. It then follows that, the ability of any particular isolate of Scedosporium to initiate infection is not determined by geographical origin or source, so all isolates appear to have the capability to start any kind of infection when the predisposing factors are in place, as previously noted. Generally, we are not able to make so many comparisons because studies dealing with the virulence of Scedosporium species are not as extensive as those of other fungal species. Moreover, some of the studies did not use the same or identical protocol. For instance, Lamaris and et al. ${ }^{16}$ used Drosophila melanogaster, contrary to our study which used mice instead. Even with that, they found that wild-type flies were resistant to infection with both S. apiospermum and S. prolificans. Recently, Gilgado et al. ${ }^{17}$ published their findings on virulence of Scedosporium species based on a new reclassification of the species and reported that S. auranticum and S. dehoogii were the most virulent of all species they screened. They also noted that $S$. dehoogii is only represented currently by five environmental strains and it is unclear whether it is able to infect humans. However, we did not include these reclassified species in our study and therefore could not compare their virulence with those of the other two species investigated. There appears to be a lot of reorganization going on in the nomenclature of Scedopsorium species. Some authors recently used a molecular identification technique to reidentify isolates in their collection and found a lot of remarkable differences. ${ }^{17}$ In general, our results correlate with clinical data because infections caused by $S$. prolificans are usually nonresponsive to antifungals and their outcomes are usually fatal compared to the sister species, S. apiospermum..$^{18-19}$ 


\section{ACKNOWLEDGEMENTS}

The authors wish to thank the several people who sent strains, especially Prof. Josep Guarro.

\section{REFERENCES}

1. Gilgado F, Cano J, Gené J et al. Molecular and phenotypic data supporting distinct species statuses for Scedosporium apiospermum and Pseudallescheria boydii and the new species Scedosporium dehoogii. J Clin Microbiol 2008; 46:766-71.

2. Gilgado F, Serena C, Cano J et al. Antifungal susceptibilities of the species of the Pseudallescheria boydii complex. Antimicrob Agents Chemother 2006; 50:4211- 3.

3. Pontón J, Rüchel R, Clemons KV et al. Emerging pathogens. Med Mycol 2000; 38(Suppl 1):S225-36.

4. Berenguer J, Diaz-Mediavilla J, Urra D, Muñoz P. Central Nervous System Infection Caused By Drosophila melanogaster: Case Report and Review. Rev Infect Dis 1989; 11:890-6.

5. De Hoog GS, Guarro J, Gené J, Figueras MJ. (2000). Atlas of Clinical Fungi, 2nd edn. Utrecht: Centraalbureau Voor Schimmelcultures/Reus: Universitat Rovigili.

6. Carrillo AJ Guarro J. In Vitro Activities of Four Novel Triazoles Against Scedosporium Spp. Antimicrob Agents Chemother 2001; 45:2151-3.

7. Gilgado F, Cano J, Gené J, Guarro J. Molecular phylogeny of the Pseudallescheria boydii species complex: proposal of two new species. J Clin Microbiol. 2005 43(10):4930-42.

8. Capilla J Guarro J. Correlation between in vitro susceptibility to voriconazole and in vivo outcome of Scedosporiosis in Guinea pigs. Antimicrob Agents Chemother 2004; 48:4009-11.
9. Fienberg R. Madura Foot in a Native American. Am J Clin Pathol 1944; 14:239-46.

10. Ajello L. The isolation of Allescheria boydii shear, an etiologic agent of mycetomas, from soil. J Trop Med 1952; 1:227-38.

11. Reyes AC. A contribution to the study of mycetoma in the Philippines: maduromycosis caused by Monosporium apiospermum (laboratory studies). Acta Med Philipp 1963; 19:89-102.

12. Schmitt JA, Zabransky RJ, Janidlo AS, Parsons JE. Experimental Maduromycosis In The Laboratory Mouse. Mycopathologia 1962; 18:164-8

13. Bell RG. Comparative Virulence and Immunodiffusion Analysis of Petriellidium boydii (Shear).Malloch Strains Isolated from Feedlot Manure and a Human Mycetoma. Can J Microbiol 1978; 24:856-63.

14. Ortoneda M, Pastor FJ, Mayayo E, Guarro J. Comparison Of The Virulence Of Scedosporium prolificans Strains From Different Origins In A Murine Model. J Med Microbiol 2002; 51:924-8.

15. Cano J, Guarro J, Mayayo E. Experimental Infection with Scedosporium inflatum. J Med Vet Mycol.1992; 30:413-20.

16. Lamaris GA, Chamilos G, Lewis RE, Kontoyiannis DP. Virulence studies of Scedosporium and Fusarium species in Drosophila melanogaster. 2007 Dec 15; 196(12):1860-4.

17. Gilgado F, Cano J, Gené J, Serena C, Guarro J. Different virulence of the species of the Pseudallescheria boydii complex. Medical Mycology 2009; 47:371-4.

18. Delhaes L, Harun A, Chen SC et al. Molecular typing of Australian Scedosporium isolates showing genetic variability and numerous S. aurantiacum. Emerg Infect Dis 2008; 14:282-90.

19. Troke $\mathrm{P}$, Aguirrebengoa K, Arteaga $\mathrm{C}$ et al. Treatment of scedosporiosis with voriconazole experience with 107 patients. Antimicrob Agents Chemother 2008; 52:1743-50. 